\title{
The VLT Laser Guide Star Facility
}

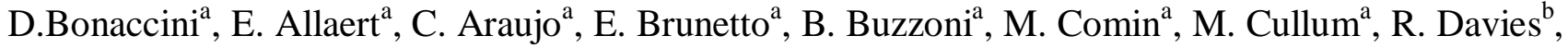

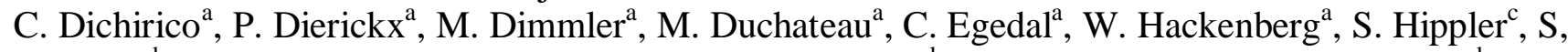

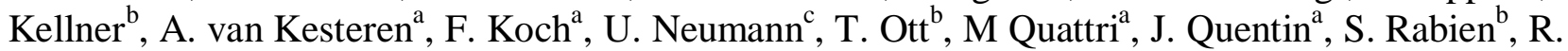 \\ Tamai $^{\mathrm{a}}$, M. Tapia ${ }^{\mathrm{a}}$ and M. Tarenghi ${ }^{\mathrm{a}}$ \\ ${ }^{a}$ European Southern Observatory, Karl-Schwarzschildstr 2, 85748 Garching, Germany \\ ${ }^{b}$ Max Planck Institut für Extraterrestrische Physik, Giessenbachstr., 85748 Garching, Germany \\ ${ }^{c}$ Max Planck Institut für Astronomie (MPA), Koenigstühl 17, 69117 Heidelberg, Germany
}

\begin{abstract}
We report on the ongoing VLT Laser Guide Star Facility project, which will allow the ESO UT4 telescope to produce an artificial reference star for the Adaptive Optics systems NAOS-CONICA and SINFONI. A custom developed dye laser producing $>10 \mathrm{~W} \mathrm{CW}$ at $589 \mathrm{~nm}$ is installed on-board of the UT4 telescope, then relayed by means of a single mode optical fiber behind the secondary mirror, where a $500 \mathrm{~mm}$ diameter lightweight, $\mathrm{f} / 1$ launch telescope is projecting the laser beam at $90 \mathrm{~km}$ altitude.

We described the design tradeoffs and provide some details of the chosen subsystems. This paper is an update including subsystems results, to be read together with our previous paper on LGSF design description ${ }^{3}$.
\end{abstract}

Keywords: adaptive optics, sodium laser guide star, large telescopes

\section{INTRODUCTION}

It has been demonstrated that AO systems can indeed work effectively with Laser Guide Stars (LGS), in the visible ${ }^{1}$ and in the NIR ${ }^{2}$. Recently the Lick Observatory has achieved K-band Strehl values of $\sim 0.6$ with LGS-AO. This technology is becoming progressively mature, and solid state lasers at $589 \mathrm{~nm}$ with adequate power and spectral format to produce LGS, are within reach in the next few years [other papers at this conference].

It is common experience that both the AO and the LGS generation facility have to be well tuned and reliable to obtain good results, in other words, good engineering of all subsystems is needed from the very start. One should avoid going over these complex systems by refurbishing them on the telescope. We have developed the LGSF based on previous systems generations' lessons learned, introducing more stability of the laser system, integration in the telescope, ease of operation and abundant diagnostics at several steps of the laser light mainstream path, with the goal of improving reliability and operations.

The strategy behind the ESO LGSF is to build a system to produce a single LGS, useful to run AO systems correcting for the Near Infra-Red bands, with the built-in provision to upgrade to multiple LGS for MultiConjugate AO. We have pushed the design, development and integration phases to complete them within a three year period, and introduced several features on the dye laser to be able to run it smoothly and without a laser specialist for extended periods of time. 
The many requirements for a laser guide star facility on the VLT telescope Yepun (UT4), have been dictated by the needs of adaptive optics, by the VLT environment and interfaces, by the Paranal Observatory policy on running instruments, and by the safety issues related to power lasers and their open-air propagation.

\section{SYSTEM HIGH LEVEL SPECIFICATION}

The Top Level Requirements for the LGSF are the following:

- LGSF is integral part of the UT4 Telescope system

- LGS has monostatic projection (i.e. on-axis of UT4 primary, launch telescope behind M2).

- The laser source is continuous-wave at 589.02nm (D2 line), $1 \mathrm{GHz}$ max linewidth

- LGS return flux $\geq 1.0 \times 10^{6} \mathrm{ph} / \mathrm{s} / \mathrm{m} 2$ @ Nasmyth focus

- LGS spot size $\leq 1.25 "$ FWHM (with $0.65 "$ seeing at $0.55 \mu \mathrm{m}, 45^{\circ}$ Zenith distance)

- LGS residual position jitter $\leq 0.05$ arcsec rms in closed loop

- LGSF is operable at UT zenith distances $\leq 60^{\circ}$,

- LGS with 20" pointing radius from UT4 centerfield

- LGSF has an embedded upgrade to 5 LGS for MCAO, up to 1 arcmin pointing radius

- Operation done by trained night assistants, with standby and on-line modes.

- General maintenance at intervals of once/week

- Safety standards implemented, Class IV lasers

- Comply with VLT Standards and the Paranal Observatory environment

\section{DESIGN OF THE LGSF}

The LGSF design solution embeds the complete laser system on the UT4 telescope, in a dedicated Class 10000 Clean Room with controlled environment. This has implied creating on board of the telescope an interferometric-quality laser environment, with optical table, thermally stabilised air, laminar flow and class 100 cleanliness in the confined volume just above the laser.

The choice of a CW laser has been done based on gains on stability, safety, LGS photon return, when compared to pulsed lasers. With the revised evaluations of the photon returns, we think that a $\mathrm{CW}$ laser gives equivalent or better photon return than any macro-micro pulse format.

The novel design of our CW dye laser was analyzed and selected only after a series of concept validation tests. The PARSEC laser is a two-stage, Master Oscillator Power Amplifier (MOPA) design, described in more detail elsewhere ${ }^{5,6}$. We had to embed several servo controls to comply with the Paranal Observatory flexible scheduling operations. The laser system has a low-power standby mode, with full power state reachable within 10 (goal) to 60 minutes. We also have 
constrained the laser design to have the laser stable and run by non-specialized operators, with the goal of regular maintenance by a specialist only once a week. Much engineering effort has been dedicated to this goal.

The laser beam is generated in a room located below the Nasmyth A focus. The beam has to be relayed to the Launch Telescope, which projects it at focussing distances between 70 and $200 \mathrm{~km}$, with an accuracy of $\pm 0.2 \mathrm{~km}$. The beam relay system is based on single mode fibers. Single mode fibers ensure diffraction limit relay up to the Launch Telescope, introduce beam losses comparable to complex mirror relays with average age coatings. It also avoids the beam quality degradation and vibration-induced pointing errors experienced by previous systems in mirror relays. The LGSF Beam Relay System (BRS) is composed of a BRS Input module, located on the PARSEC laser table, a single mode fiber, and a BRS Output module, attached to the Launch Telescope.

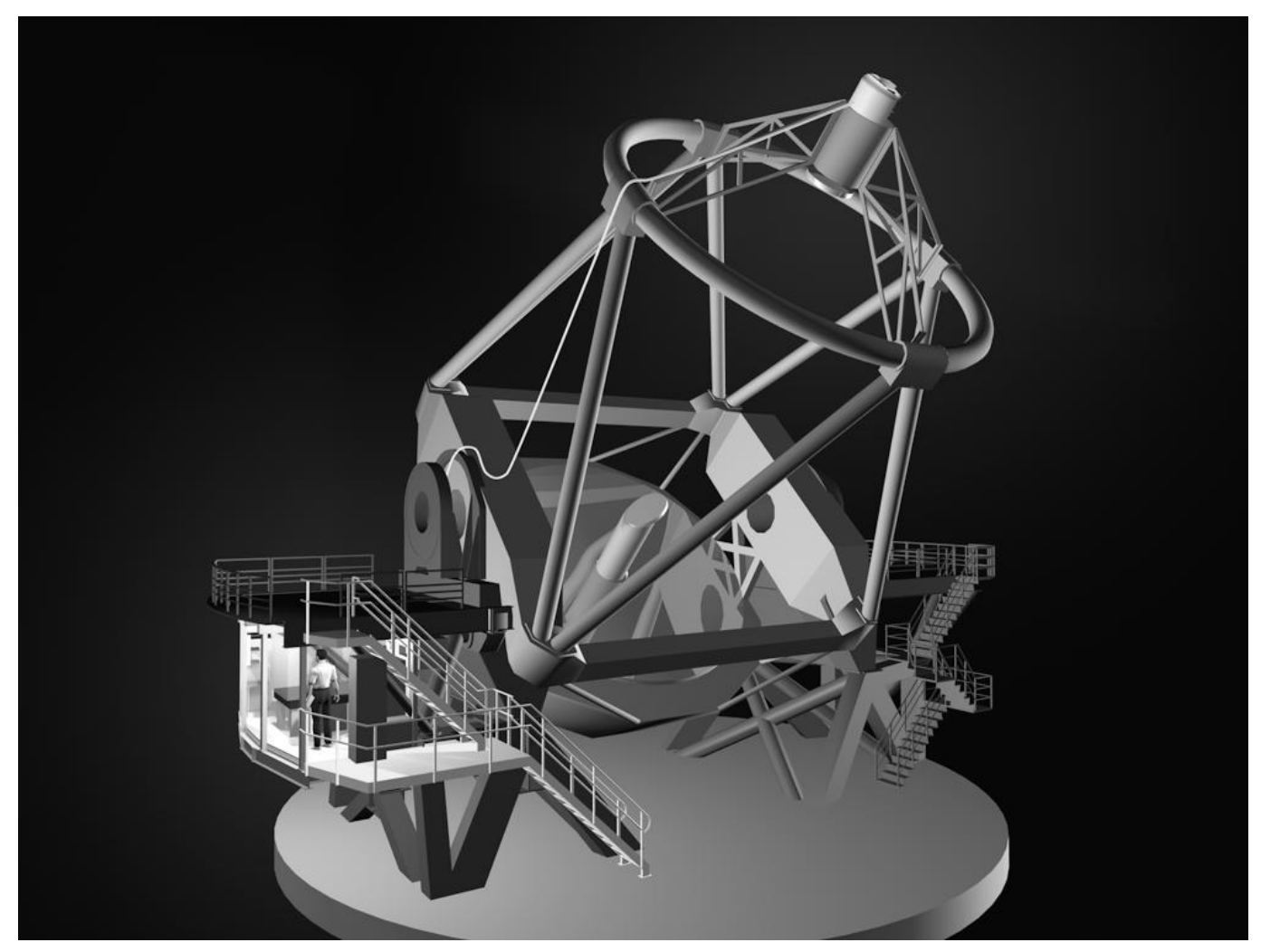

Figure 1: The LGSF, with the Laser Clean Room located underneath the UT4 Nasmyth A platform, the fiber relay and the Launch Telescope above UT4 M2.

The BRS Input module formats spectrally (yielding 4 single frequency lines equally spaced within a $500 \mathrm{MHz}$ envelope) and spatially the PARSEC beam, using an Electro-Optical modulator and an optimized fiber-coupling lens. Four beam stabilization servo-controls avoid the effect of transients during low-high power transitions, keeping the laser beam steady. The BRS Input module measures on line: the laser beam power, the beam quality and spectral format of the incoming PARSEC beam, as well as the power, Stimulated Brillouin Scattering, polarization, beam quality, and spectral format of the laser beam entering the fiber relay.

The $27.5 \mathrm{~m}$ long single mode fiber used for the fiber relay has to stand $10 \mathrm{~W} \mathrm{CW}$ in the single mode core. To avoid the onset of known non-linear effects, such as the Stimulated Brillouin Scattering (SBS), a $15 \mu \mathrm{m}$ core Photonic Crystal Fiber is used, 
as well as a spectral broadening of the laser line. A sturdy, protected fiber cable with embedded 4 additional fiber spares is used, to go through the altitude cable wrap of the Nasmyth A and on the shortest path to the Launch Telescope located behind M2.

The f/12.5 fiber output (5\% gaussian beam level) is on the Beam Relay System Output module, attached to the Launch Telescope (LT). The fiber is on the focal plane of the beam collimator optics, which produces the $40 \mathrm{~mm}$ parallel beam at the input of the LT. For the diagnostic devices, $1 \%$ of the laser beam is sampled. The fiber output is on a motorized stage, which is used for pointing the Laser Guide Star (LGS) within a 20" radius from the UT4 optical axis. The LGS jitter is corrected by moving the fiber output with a Physik Instrumente nanocube piezo actuator system. The correcting signal comes from the client Adaptive Optics System, and is filtered in the LGSF electronics.

The diagnostic devices embedded in the BRS output measure beam quality, polarization, power of the beam coming from the fiber, as well as of the return beam coming beck from the last surface of Launch Telescope exit window.

The f/1.03 Launch Telescope is a $12.5 \mathrm{X}$ beam expander with $50 \mathrm{~cm}$ exit beam, made of Carbon Fiber Reinforced Plastic (CFRP) and lightweighted Zerodur, inserted in an aluminium housing.

More details on the description of the LGSF are contained in our previous SPIE conference paper ${ }^{3}$. We will focus on this paper on the subsystems technical details, on the safety system design, and on the project status.

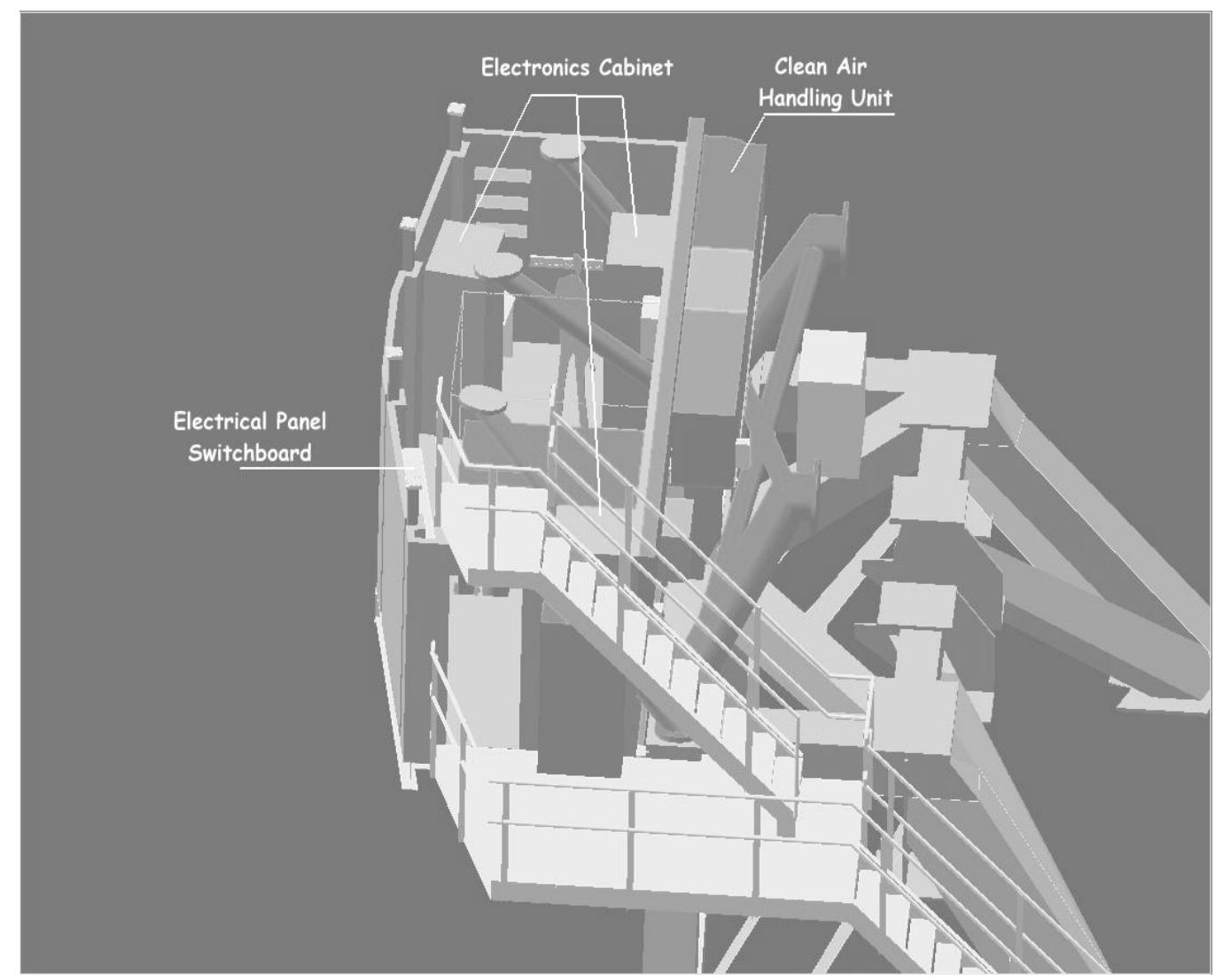

Figure 2: Design scheme of the LGSF-Laser Clean Room. The Nasmyth A platform above it is not shown. All LGSF electronic racks as well as the PARSEC laser are in the room. 


\section{THE LASER CLEAN ROOM}

The laser clean room (LCR) has now been mounted on VLT-UT4 in Paranal, together with its fire-fighting system, clean room devices and numerous safety interlocks. It was built by Daldrop -Ing Hüber Gmbh. $25 \mathrm{~kW}$ of electrical power and of correspondingly cooling power have been provided, together with compressed air, LAN and telephone lines. The LCR has been specified to be resistant to earthquakes, which has driven the design and the choice of the components. Quasi-static 5g specification has been followed for the sub-components.

With accelerometers, we have verified that there is negligible contribution from the LCR air handling unit fans to the vibrations on the Nasmyth platform above. This has been obtained by carefully introducing adequate damping. We have also verified that our specifications of $\pm 1.5^{\circ} \mathrm{C}$ difference of the LCR outer surface with respect to ambient temperature are matched. In the spring of 2003 we will install in the LCR three electronic cabinets, the PARSEC optical table with its covers and laminar flow system, and all electrical cables and cooling pipes for the various subsystems.

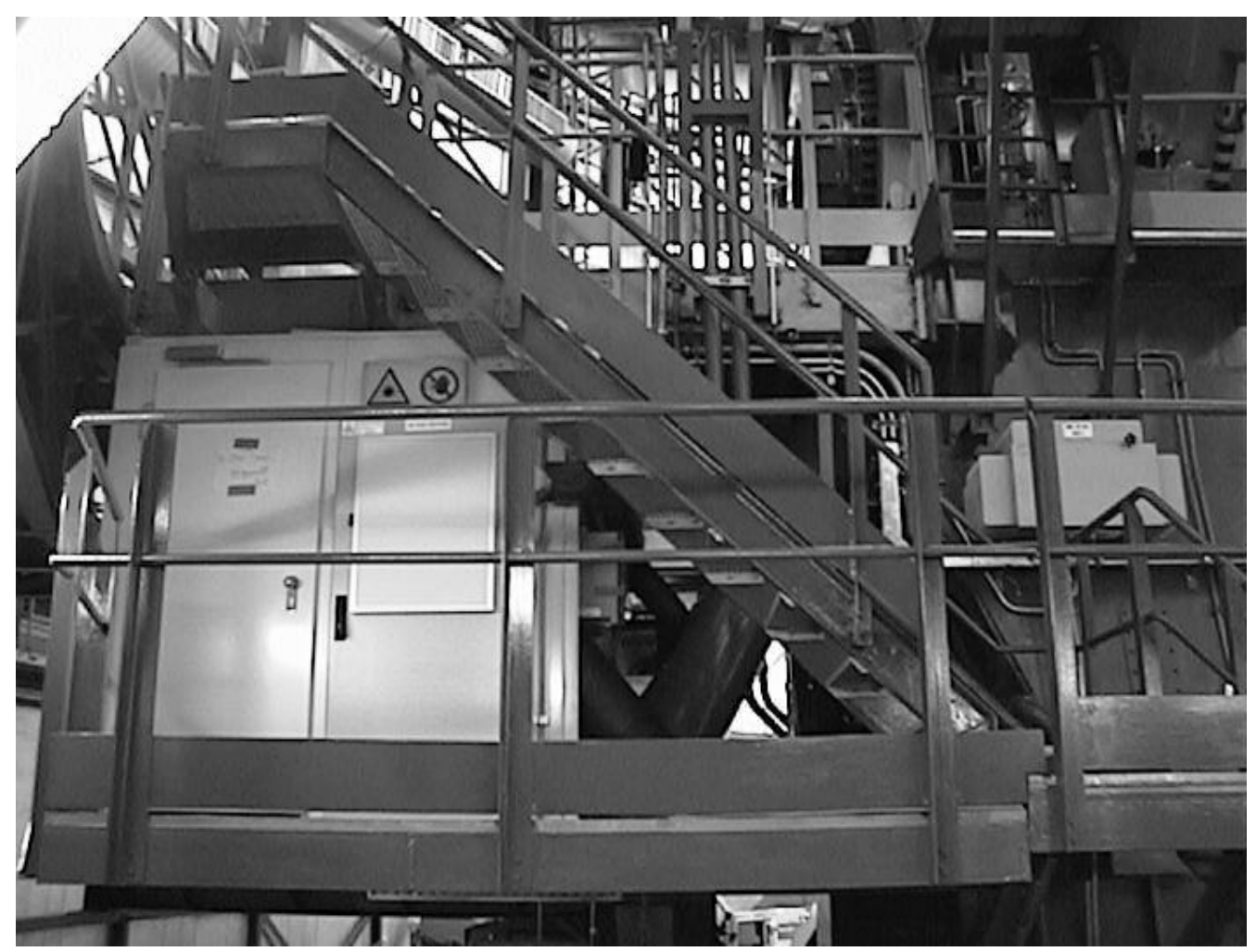

Figure 3: LCR side view, mounted on board of VLT-UT4 under the Nasmyth A platform. The coded access door and the air handling unit control cabinet are seen in this picture.

\section{THE PARSEC LASER}

The PARSEC CW dye laser, a Master Oscillator-Power Amplifier design with free-jet high pressure dye flow, has been able to demonstrate a power output of $12.6 \mathrm{~W} \mathrm{CW}$ when pumping the dye with an $\mathrm{Ar}^{+}$laser. 
Conversion efficiencies $>40 \%$ had been predicted following our first tests at Calar Alto in $2000^{4}$. Up to $42 \%$ conversion efficiency in PARSEC (in a low-loss cavity) has more recently been demonstrated when pumping Rhodamine $6 \mathrm{G}$ at $532 \mathrm{~nm}$ with Coherent Verdi V10 lasers ${ }^{5}$. The required $589 \mathrm{~nm}$ full power output is $10 \mathrm{~W} \mathrm{CW}$, now the expected power output is more than $16 \mathrm{~W} \mathrm{CW}$, i.e. above specifications, as indicated in Figure 4. More results on the PARSEC subsystem are reported in two other papers at this conference ${ }^{6,7}$.

Several servo-controls keep the Master Oscillator tuned to the Sodium D2 line, the Power Amplifier locked cavities and the laser beams stable in alignment ${ }^{6}$.

Recently the servo control for the frequency tuning on the Master Oscillator has been locked within $\pm 1 \mathrm{MHz}$ to the center of the Na-D2 line, while producing up to $3 \mathrm{~W} \mathrm{CW}$ with a beam quality factor $\mathrm{M}^{2}<1.05$, and a Verdi V10 pump of 10W CW.

The 5 Verdi V10 pump beams are internally stabilized in jitter by servo-controls, as well as the beam relay from the Master Oscillator to the Power Amplifier?

The anastigmatic Power Amplifier cavity is now stabilized with a double-wavelength servo-control, using a fast $(10 \mathrm{KHz})$ piezo-control loop. This has been tested to avoid mode-hopping. While seeded by the Master oscillator, the two free dye-jets in the Power Amplifier are pumped at 532nm with 20W $\mathrm{CW}$ pump power at $532 \mathrm{~nm}$ for each dye jet. The conversion efficiency has been tested, and the output power reached $12.6 \mathrm{~W}$ $\mathrm{CW}$ using an Argon ion pump laser. In more recent tests ${ }^{6}$ with 2 Verdi V10 lasers pumping the jets in the amplifier, an output power of $10.5 \mathrm{~W}$ was reached (of which the seed power form the Master was only $1.9 \mathrm{~W}$ ). When the amplifier is pumped with 4 Verdi V10 lasers, there will be a large margin of safety with regards to output power.

The required PARSEC stand-by and operation modes, with 10 min goal switching between the two, will be achieved by keeping the master oscillator running at low power at all times, and progressively switching on the power amplifier pumps only for full operation. This procedure and the fact that all needed servo-controls are kept running at all times, ensure in our opinion that the PARSEC laser can switch smoothly from low to high powers (standby to full operation modes), and that it can be run effectively with weekly maintenance periods. We have still to verify this assumption in the coming months. The final acceptance of the PARSEC laser is foreseen in May 2003. 


\section{THE BEAM RELAY SYSTEM}

The Beam Relay system has three sections, the Beam Relay Input located on the PARSEC optical table and already described $^{3}$, the single mode Fiber relay and the Beam Relay Output module.

\subsection{THE BEAM RELAY SYSTEM INPUT}

We are currently assembling and testing the Beam Relay Input system. We have custom built the Electro-Optic modulator and its control electronics, where we will achieve phase shifts of 2.1-2.5 rad. We have custom built and tested microprocessor-based alignment control units, based on Position Sensitive Devices and Physik Instrumente Tip-Tilt stages, controlled over CAN-Bus. Two "rock-solid" optical beams are created in the BRS Input, one for the crystal EO modulator and one for the fiber input. We found it is very critical to ensure that the high power laser beam is of good quality $\left(\mathrm{M}^{2}<1.3\right)$, precisely and stably focussed on the $15 \mu \mathrm{m}$ fiber input, with a jitter contained within $\pm 0.1 \mu \mathrm{m}$.

\subsection{THE FIBER RELAY}

For the fiber relay, after much testing during the last year we have decided to use the Photonic Crystal fibers (PCF) from Crystal Fiber AG. PCF allow having Large Effective Area cores and to transfer higher power densities, still being single mode. The LGSF fiber specifications allowing transferring $10 \mathrm{~W} \mathrm{CW}$ of laser power are:

$\begin{array}{ll}\text { Core material: } & \text { Pure Silica core, UV grade, treated for pulling losses } \\ \text { Core diameter: } & 15 \pm 1 \mu \mathrm{m} \\ \text { Cladding diameter: } & 125 \pm 5 \mu \mathrm{m} \\ \text { Coating diameter: } & 245 \pm 10 \mu \mathrm{m} \\ \text { Coating material: } & \text { Acrylate } \\ \text { Length: } & 27.5 \mathrm{~m} \\ \text { Bulk attenuation: } & <10 \mathrm{~dB} / \mathrm{km} \text { at } 589 \mathrm{~nm} \\ \text { Macrobending losses: } & <1 \mathrm{~dB} / \mathrm{km} \text { at } 589 \mathrm{~nm} \text {, with } \mathrm{R} \geq 0.25 \mathrm{~m} \text { bending radius } \\ \text { Microbending losses: } & <5 \mathrm{~dB} / \mathrm{km} \text { at } 589 \mathrm{~nm}\end{array}$

We are in progress to meet all the requirements, in a genuine collaborative effort with the producer. The measured macrobending losses are $<0.2 \mathrm{~dB} / \mathrm{km}$, while the combination of microbending and bulk losses is measured $<15 \mathrm{~dB} / \mathrm{km}$.

Given that the fiber loss parameters have recently been met, we are working on the experimental optimization of the fiber coupling parameters. It seems critically important for the coupling efficiency, to match the fiber N.A.

Photonic crystal fibers have only recently (June 2001) started to be produced commercially. They exhibit unusual properties when compared to standard step-index fibers, for example the Numerical Aperture is linearly dependent on wavelength, the mode field diameter is constant with wavelength, the macro and micro bending losses vary with wavelength in an unusual way, the pulling method can create losses etc. Although these new properties have been somewhat modeled theoretically, the effect of fabrication tolerances has not and therefore the experimental tests are a time-consuming must for us, which is being tackled.

To transfer from PARSEC more than $10 \mathrm{~W}$ CW of laser power (which is indeed our specification) we will at a second stage of LGSF either use a larger PCF, or more fibers recombined, or follow the development of hollow-core PCF. 


\subsection{THE BEAM RELAY SYSTEM OUTPUT}

The functional layout of the fiber output is shown in Figure 5 and described in its caption.

As the BRS output is attached to the Launch Telescope and enclosed behind M2, we have embedded much remote beam diagnostic devices. The BRS output optics, as well as the Launch Telescope, is air tight enclosed under dry $\mathrm{N}_{2}$ atmosphere to avoid fogging and to retard the oxidation of the coatings. Much attention has been paid to the system stiffness to avoid high frequency jittering of the LGS, outside the bandwidth of the Adaptive Optics system.

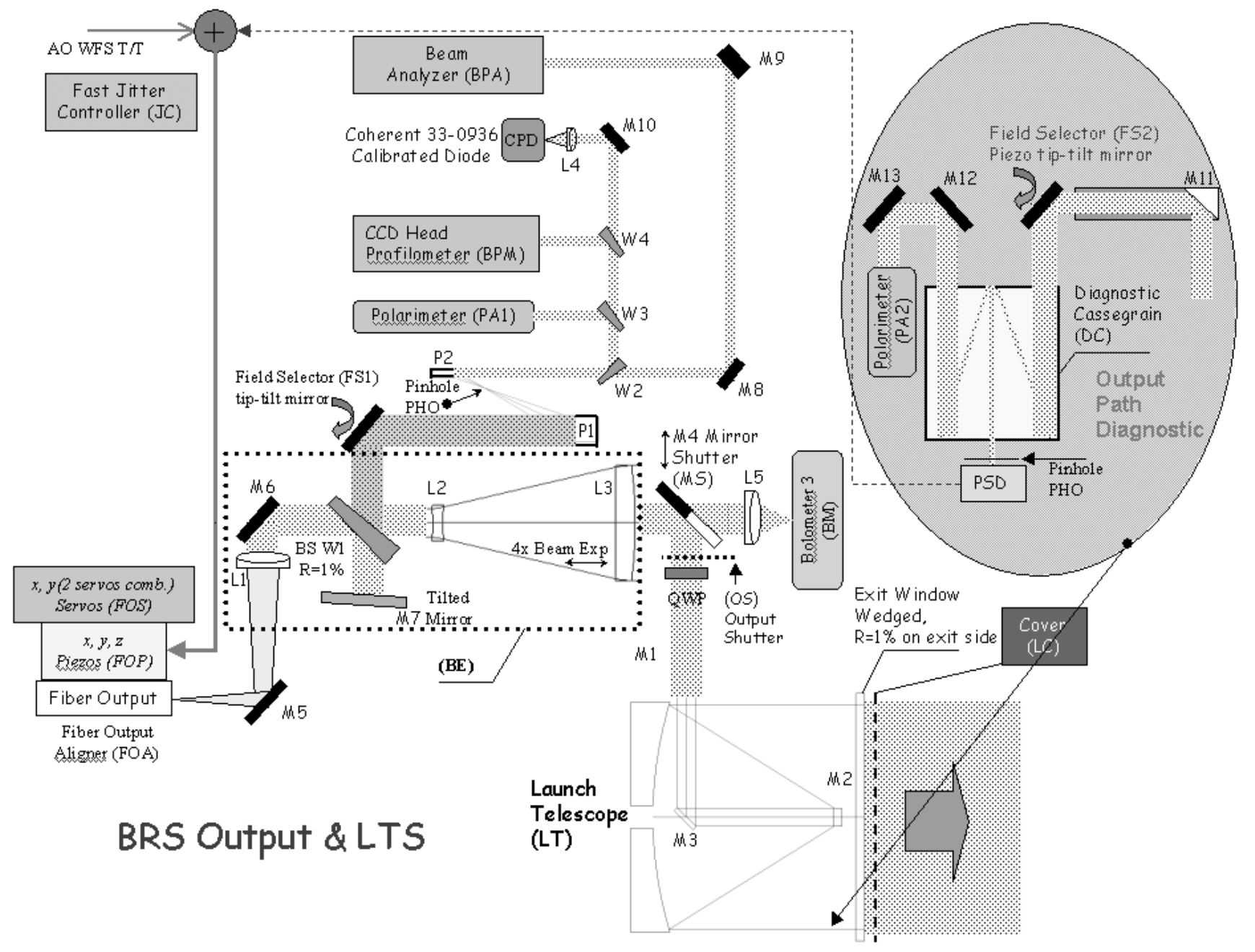

Figure 5: The BRS output optomechanics allows to sample forward and backward laser beams for diagnostics, using the BSWI beam splitter, inserted on a $10 \mathrm{~mm}$ parallel beam produced by L1. The backward laser beam is created by the last surface of the exit window, which retroreflects $1 \%$ of the laser power. A Field selector (FS1) and a $3 X$ beam reducer with a pinhole and two off-axis parabolas $(P 1-P 2+P H O)$ allow to choose between forward and backward beams to diagnose. A $3.3 \mathrm{~mm}$ beam is directed via W2-W4 and M8-M10 to the diagnostic devices, to measure beam quality (BPA), polarization states (PA1), beam profile (BPM) and relative power. After BS W1, a second stage $4 X$ beam expander L2-L3, creates the $40 \mathrm{~mm}$ parallel beam to input the $500 \mathrm{~mm}$ Launch Telescope. A movable mirror shutter (M4) can direct the beam to The $500 \mathrm{~mm}$ diameter outgoing beam is sampled by the output path diagnostics (see inset) which measures polarization (PA2), relative power and beam direction via a Position Sensitive Device (PSD). 


\section{THE LAUNCH TELESCOPE}

The Launch Telescope top level requirements are:

- weight below $70 \mathrm{~kg}$;

- useful diameter $500 \mathrm{~mm}, 1 / \mathrm{e}^{2}$ intensity at $360 \mathrm{~mm}$ diameter;

- overall length below $650 \mathrm{~mm}$, including entrance window and supports;

- diffraction limited optical quality (60 $\mathrm{nm}$ rms wavefront error transmitted);

- first resonant frequency $>120 \mathrm{~Hz}$;

- operation up to $60 \mathrm{deg}$ from Zenith, up to $50 \mathrm{~W} \mathrm{CW}$ power densities

- Removable laser quality optical coatings without the need to repolish the optics

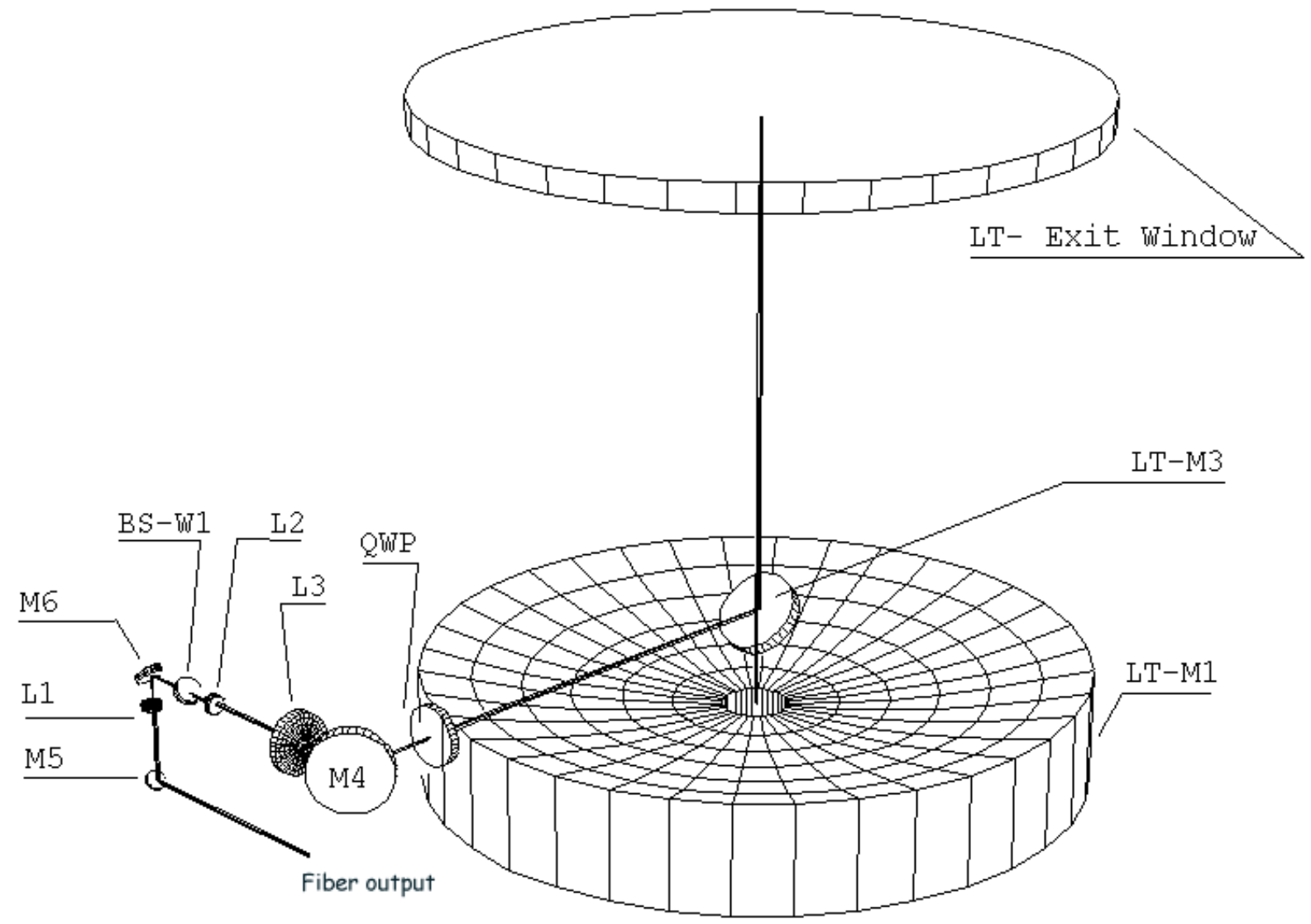

Figure 6: The path of the laser beam from the fiber output through the BRS Output optics, to the launch Telescope is shown. The Launch Telescope System is comprised by the entrance window QWP, M1 to M3 and the Exit Window.

The Launch Telescope is at the moment in fabrication at Galileo Avionica. Delivery is scheduled in April, 2003. The design has eventually shown to meet the requirements, except for an excess weight of $2 \mathrm{~kg}$. Dynamical and static analyses have 
been performed to verify compliance with requirements. It has light-weighted Zerodur optics, mounted on a Carbon Fiber Reinforced Plastics (CFRP) structure, which gives a very high rigidity with lightweight. The chosen CFRP type does not suffer from thermal or hygroscopic problems. The f/1.03 optics is a confocal Cassegrain design, which has a nominal performance of Strehl $>0.99$ at all field position within 1 arcmin radius. The flexure and tolerance error budgets are very demanding on the design, fabrication and testing.

\section{SAFETY ASPECTS}

It should not be underestimated the engineering effort which goes into the safety measures of Laser Guide Star systems. There are strict regulations for lasers, besides Observatory regulations to follow, to avoid accidents.

We have chosen to follow for the laser safety aspects the ANSI standards Z136.1 and Z136.6, since after comparison with the European Norms EN60825 it turns out that they are more up to date for our application - although with time the picture may change.

There is a very large set of interlock and interlocking conditions for LGSF, which have been studied in great detail via an extensive Hazard Analysis, with up to three level of concurrency, and reported in the LGSF design documents. We give hereafter only some of the main functionalities, the reader interested to receive them or to know more details should contact Carsten Egedal (cegedal@eso.org). The scheme is shown in the following figure

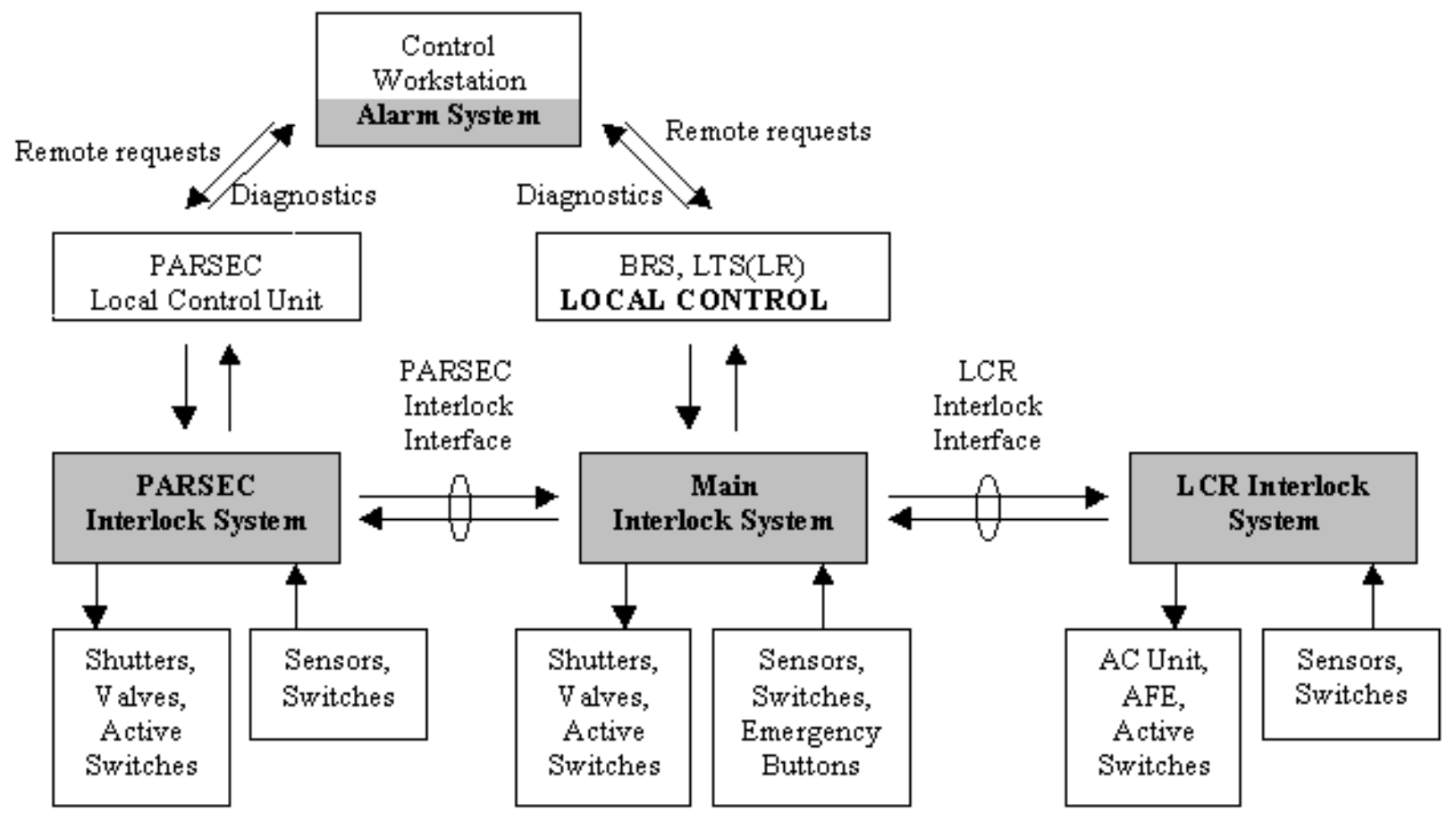

The PARSEC dye laser is installed in the Laser Clean Room (LCR), placed underneath the Nasmyth A platform of UT4. The room hosts the laser, part of the Beam Relay and Diagnostic system, all LGSF electronics cabinets, all the electronics of the laser pumps and the interlock electronics. 
The Laser Clean Room is equipped with an automatic fire-fighting system built by Total Walter, based on INERGEN gas spread by nozzles and triggered by smoke-flame sensors placed in the room and inside the PARSEC laser optical table. INERGEN has the great advantage of being breathable (at least for a few minutes), as opposed to $\mathrm{CO}_{2}$.

All electrical cables and materials used for the LCR are non flammable, the floor is antistatic and careful grounding avoids static discharges.

The Rhodamine 6G laser dye is normally diluted in Ethylene Glycol, which is hardly flammable at normal temperatures. Alcohol sensors are located close to the dye pump, which in case of spillage trigger interlocks on the laser dye pump and on the Coherent Verdi laser pumps.

An Earthquake sensor is interlocked with the shut-off of the laser system and the dye pumps.

An oxygen sensor interlocks with an automatic LCR ventilation system, and is active during normal operations (not during fire alarms!).

Liquid spillage sensors are interlocked to the liquid coolant and the dye solution circuits; the latter is interlocked to the pump lasers.

Room access is limited to trained personnel only, via a coded key at the door, which may be overridden in exceptional cases. Room exit is unrestrained.

The LCR is a Class I laser controlled area, as long as the laser beam path is shielded in the PARSEC and BRS optomechanical modules. During laser maintenance the service access panels of the modules may be removed and their switches will trigger the laser shutter as well as an external warning light, since the LCR becomes a Class IV laser environment.

The laser beam power is monitored along the beam relay system, including the exit of the fiber relay, and interlocks will block the PARSEC laser shutter in case of unexpected beam relay losses.

Watchdogs between the LGSF electronics control units and the Workstation will stop the laser operation in case of loss of communication between computers.

Two aircraft detection cameras mounted on the UT4 top ring and operating in the visible will interlock the laser propagation as long as the aircraft is within 70 degrees field of view.

Stop buttons in the LCR and on the UT4 Azimuth platform will allow to remove all power, or to shut the laser propagation, respectively.

\section{CONCLUSIONS}

The LGSf project is more than half way on its three years of duration.

The LGSF Final Design Review has been passed, all major subsystems contracts are in place and we are starting to assemble subsystems.

The first of two steps of integration on the telescope has been completed.

The summer-fall 2003 will see the crucial installation and the first test time for the LGSF. So far we are confident to proceed on the scheduled path in time. 


\section{REFERENCES}

1. R.Q.Fugate et al: Two generations of laser-guide-star Adaptive Optics experiments at the Starfire Optical Range, JOSA A, Vol. 11, pp. 310-325, 1994

2. D.Gavel et al.: Recent Science and Engineering Results with the Laser Guidestar Adaptive optics System at Lick Observatory, contained in this proceedings

3. D.Bonaccini et al.: ESO VLT Laser Guide Star Facility, in SPIE Proc. of the International Symposium on Adaptive Optics Systems Technologies II, Vol 4494, pp.276-289, 2001, R.Tyson et al. Eds

4. D. Bonaccini, W. Hackenberg, R.Davies, S. Rabien and T. Ott: VLT Laser Guide Star Facility: First Successful Test of the Baseline Laser Scheme, The ESO Messenger no. 100, Dec 2000 - available also online at http://www.eso.org/genfac/pubs/messenger

5. S. Rabien, R. Davies, T. Ott, S. Hippler, U. Neumann: PARSEC, the Laser for the VLT, in SPIE Proc. of the International Symposium on Adaptive Optics Systems Technologies II, Vol 4494, pp.325, 2001, R.Tyson et al. Eds

6. S. Rabien, R. Davies, T. Ott, J. Li, S. Hippler, U. Neumann, Design of PARSEC, the VLT Laser, this proceedings

7. R. Davies, T. Ott, J. Li, S. Rabien, U. Neumann, S. Hippler, D. Bonaccini, W. Hackenberg, Operational Issues for PARSEDC, the VLT Laser, this proceedings 